\title{
ON THE HEIGHT OF THE FIRST STIEFEL-WHITNEY CLASS
}

\author{
HOWARD L. HILLER
}

\begin{abstract}
Let $G_{k}\left(\mathbf{R}^{n+k}\right)$ denote the grassmann manifold of $k$-planes in real $(n+k)$-space and $w_{1} \in H^{1}\left(G_{k}\left(R^{n+k}\right) ; Z_{2}\right)$ the first Stiefel-Whitney class of the universal bundle. We determine, for many $(k, n)$, the exact height of $w_{1}$ in the cohomology ring. We also indicate the combinatorial significance of the complex analogue of these computations.
\end{abstract}

Let $G_{k}\left(\mathbf{R}^{n+k}\right)$ denote the grassmann manifold of $k$-planes in real $(n+k)$-space. We have previously determined estimates on the height of the first Stiefel-Whitney class $w_{1}$ of the universal $k$-plane bundle over $G_{k}\left(\mathbf{R}^{n+k}\right)$ [2]. Indeed, we showed that if $1<k<n$ and $2^{s}<n+k<2^{s+1}$, then the height of $w_{1}$ is $2^{s+1}-\alpha$, where $\alpha$ is either 1 or 2 (unless $k=2^{t}=n$, in which case we can only assert $1<\alpha<2^{t}$ ). As the codimension $n$ increases, it is clear that the height of $w_{1}$ is nondecreasing; hence it suffices to find where in the intervals of $n:\left[2^{s}+1-k, 2^{s+1}-k\right]$ the value of $\alpha$ jumps down. For $k=2$, it was shown that $\alpha$ is identically 2 [2]. In this note, we settle the problem for $k=3,4,5$ and for $k>6$ (except for finitely many $n$ ).

We use the Schubert calculus description of the mod 2 cohomology of $G_{k}\left(R^{n+k}\right)$ [1]. Additively, a $Z_{2}$-basis is provided by the Schubert symbols $\left(a_{1}, \ldots, a_{k}\right)$ where $0<a_{1}<\ldots<a_{k}<n$. Indeed, the tangential and normal Stiefel-Whitney classes of the universal bundle are themselves represented by Schubert symbols; namely

$$
w_{i}=(0, \ldots, 0, \underbrace{1, \ldots 1}_{i}), \quad \bar{w}_{j}=(0, \ldots, 0, j),
$$

where $1<i<k, 1<j<n$. One often refers to the $\bar{w}_{j}$ as special Schubert symbols. They are special, from one point of view, since there is a combinatorial formula describing their multiplication by an arbitrary Schubert symbol; namely

$$
\bar{w}_{j}\left(a_{1}, \ldots, a_{k}\right)=\sum\left(b_{1}, \ldots, b_{k}\right),
$$

where the sum ranges over all $k$-tuples $\left(b_{1}, \ldots, b_{k}\right)$ such that $a_{i}<b_{i}<a_{i+1}$, $1<i<k$ (where $a_{k+1}=n$ ) and $b_{1}+\cdots+b_{k}=j+a_{1}+\cdots+a_{k}$. This is called the Pieri intersection formula. We now begin with

LEMMA 1. In $H^{*}\left(G_{3}\left(\mathbf{R}^{n+3}\right) ; \mathbf{Z}_{2}\right)$,

$w_{1}^{j}=\sum_{p, q}\left[\left(\begin{array}{c}p+q-1 \\ q\end{array}\right)\left(\begin{array}{c}j \\ p+q-2\end{array}\right)+\left(\begin{array}{c}p+q+1 \\ q+1\end{array}\right)\left(\begin{array}{c}j+1 \\ p+q\end{array}\right)\right](p, q, j-p-q)$.

Proof. Use the Pieri formula and induction; see [3].

Received by the editors December 4, 1978.

AMS (MOS) subject classifications (1970). Primary 55C30. 
LEMMA 2.

$$
\left(\begin{array}{c}
2^{s}+j \\
k
\end{array}\right) \equiv 0 \quad(\bmod 2) \quad \text { if } j+1<k<2^{s}-1
$$

Proof. The result is immediate for $j=0$; we then invoke induction and

$$
\left(\begin{array}{c}
2^{s}+j \\
k
\end{array}\right)=\left(\begin{array}{c}
2^{s}+j-1 \\
k
\end{array}\right)+\left(\begin{array}{c}
2^{s}+j-1 \\
k-1
\end{array}\right) \equiv 0(\bmod 2)
$$

for $k>j+1$. This completes the proof.

It is now easy to show

Proposition 3. In $H^{*}\left(G_{3}\left(\mathbf{R}^{2^{+}+2}\right) ; \mathbf{Z}_{2}\right)$,

$$
w_{1}^{2^{s+1}-1}=\sum_{i=1}^{2^{s-1}}\left(i, 2^{s}-i, 2^{s}-1\right) \neq 0 .
$$

Proof. By Lemma 1, this is equivalent to

$$
\left(\begin{array}{c}
p+q-1 \\
q
\end{array}\right) \equiv 0 \quad(\bmod 2)
$$

unless $p+q=2^{s}$ (for which clearly the binomial coefficient is odd). By the Schubert condition, $q<2^{s+1}-1-p-q$, i.e. $q<2^{s}-(p+1 / 2)$. Also $p+q>$ $2^{s}+1$, or $q>2^{s}-p+1$; so we can write $q=2^{s}-(p-1)+j, 0<j<(p-$ 1/2). Reindexing by $j$, we see

$$
\left(\begin{array}{c}
p+q-1 \\
q
\end{array}\right)=\left(\begin{array}{c}
2^{s}+j \\
2^{s}-(p-1)+j
\end{array}\right)
$$

By Lemma 2, it suffices to check that $j+1<2^{s}-(p-1)+j<2^{s}-1$. The first inequality becomes $p<2^{s}$ which is immediate from the Schubert condition. The second inequality asserts $j+2<p$. So when $j$ is maximized this becomes $p>3$. This is also immediate, since if $p<3$, it is easy to check that $p+q=2^{s}$. This completes the proof.

In [2, Lemma 4.6] we showed $w_{1}^{2^{++1}-1}=0$ in $H^{*}\left(G_{3}\left(\mathbf{R}^{2^{3}+1}\right) ; \mathbf{Z}_{2}\right)$, so that for $k=3, \alpha=2$ at the first place in the interval and by Proposition $3, \alpha=1$ thereafter. To settle the case $k=4$ we have

Proposition 4. For $s>3$, in $H^{*}\left(G_{4}\left(\mathbf{R}^{2^{3}+1}\right) ; \mathbf{Z}_{2}\right)$,

$$
w_{1}^{2^{s+1}-1} \neq 0 \text {. }
$$

Proof. By Propositions 3.1 and 3.2 of [2], $w_{1}^{2 s}=\left(1,1,1,2^{s}-3\right)$. So it suffices to compute the height of $w_{1}$ inside of $H^{*}\left(G_{3}\left(\mathbf{R}^{2^{3}-1}\right) ; \mathbf{Z}_{2}\right)$. Now we have the inclusion

$$
i: G_{3}\left(\mathbf{R}^{2^{s-1}+2}\right) \subset G_{3}\left(\mathbf{R}^{2^{s-1}}\right) \text {. }
$$

In the former space, by Proposition $3, w_{1}^{2^{s}-1} \neq 0$; hence also in the latter space. It is easy to see there is an injection

$$
e: H^{*}\left(G_{3}\left(\mathbf{R}^{2^{s}-1}\right) ; \mathbf{Z}_{2}\right) \rightarrow H^{*}\left(G_{4}\left(\mathbf{R}^{2^{s}+1}\right) ; \mathbf{Z}_{2}\right)
$$


defined on the Schubert basis by

$$
e\left(a_{1}, a_{2}, a_{2}\right)=\left(a_{1}+1, a_{2}+1, a_{3}+1,2^{s}-3\right) .
$$

Graphically, we embed the 3-cycle in the upper left-hand corner

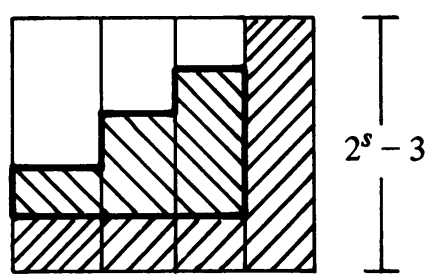

where the heights of the column indicate the entries of the Schubert cycle. The Pieri formula then implies

$$
w_{1}^{2^{5}+x}=e\left(w_{1}^{x}\right)
$$

where the locations of the $w_{1}$ 's are determined by context. Since $e$ is injective and $w_{1}^{2^{2}-1} \neq 0$, we conclude

$$
w_{1}^{2^{s+1}-1}=w_{1}^{2^{s}+2^{s}-1}=e\left(w_{1}^{2^{s}-1}\right) \neq 0 .
$$

Proposition 4 immediately yields that $\alpha$ is identically 1 for the case $k=4$.

Proposition 5. For $s \geq 3$, in $H^{*}\left(G_{5}\left(\mathbf{R}^{2^{s}+1}\right) ; \mathbf{Z}_{2}\right)$,

$$
w_{1}^{2^{s+1}-1} \neq 0 \text {. }
$$

Proof. The argument is entirely analogous to that of Proposition 4, except we use the inclusion

$$
G_{4}\left(\mathbf{R}^{2^{-1}+1}\right) \subset G_{4}\left(\mathbf{R}^{2^{2}-1}\right) .
$$

Hence, we must assume $s \geq 4$. For the case $s=3$, we observe $G_{5}\left(R^{9}\right) \simeq G_{4}\left(R^{9}\right)$, so the result again follows from Proposition 4.

Finally, we come to

Proposition 6. For $s>k-2$, in $H^{*}\left(G_{k}\left(\mathbf{R}^{2^{s}+1}\right) ; \mathbf{Z}_{2}\right)$,

$$
w_{1}^{2^{s+1}-1} \neq 0 \text {. }
$$

Proof. Use the inclusion $G_{k-1}\left(\mathbf{R}^{2^{s-1}+1}\right) \subset G_{k-1}\left(\mathbf{R}^{2^{3}-1}\right)$ and induction.

We summarize our results in the following table:

\begin{tabular}{c|c|c|c|c|c}
\hline$k$ & $2^{s}+1-k$ & $2^{s}+2-k$ & $\cdots$ & $2^{s+1}-1-k$ & $2^{s+1}-k$ \\
\hline$k=2$ & 2 & 2 & $\cdots$ & 2 & 2 \\
\hline$k=3$ & 2 & 1 & $\cdots$ & 1 & 1 \\
\hline$k=4$ & 1 & 1 & $\cdots$ & 1 & 1 \\
\hline$k=5$ & 1 & 1 & $\cdots$ & 1 & 1 \\
\hline$k>6$ & 1 & 1 & $\cdots$ & 1 & 1
\end{tabular}

$(s>k-2)$

Values of $\alpha(k, n)$, where $k<n$, i.e. $k<2^{s-1}$; height of $w_{1}=2^{s+1}-\alpha(k, n)$ in the $\bmod 2$ cohomology of $G_{k}\left(\mathrm{R}^{n+k}\right)$. 
Remark. One can ask similar questions for the first Chern class $c_{1}$ in $H^{2}\left(G_{k}\left(C^{n+k}\right) ; Z\right)$. Of course, the integral version of the Pieri formula immediately implies that $c_{1}^{n k} \neq 0$. (One could also argue that $c_{1}$ is a Kähler 2-form, so raising it to the complex dimension of the manifold is a volume element.) Now the $2 n k$ cohomology group of $\mathrm{C}^{n+k}$ is generated by the fundamental class $(n, n, \ldots, n)$; so $c_{1}^{n k}=N(n, \ldots, n)$ for some integer $N$. It is an immediate consequence of the "hook formula" that

$$
N=\frac{(n k) !}{1^{1} 2^{2} \cdot \ldots \cdot k^{k}(k+1)^{k} \cdot \ldots \cdot n^{k}(n+1)^{k-1} \cdot \ldots \cdot(n+k-1)^{1}} .
$$

The number on the right can also be interpreted as the degree of a certain irreducible representation of the symmetric group on $n k$ letters. It might be interesting to try a similar computation for $G / P$, where $G$ is a complex Lie group and $P$ is a maximal parabolic.

Finally, note that if $k=2, N$ is the $n$th Catalan number $(n+1)^{-1}\left(\begin{array}{c}2 n \\ n\end{array}\right)$.

\section{REFERENCES}

1. S. Chern, On the multiplication in the characteristic ring of a sphere bundle, Ann. of Math. (2) 49 (1948), 362-372.

2. H. Hiller, On the cohomology of real grassmannians, Trans. Amer. Math. Soc. 257 (1980), 521-533.

3. V. Opriou, Some non-embedding theorems for the grassmann manifolds, $G_{2, n}$ and $G_{3, n}$, Proc. Edinburgh Math. Soc. 20 (1977), 177-185.

Mathematical InstTtute, OXPORd Universtty, OXPord OX1 3LB, ENGLAND

Current address: Department of Mathematics, Yale University, New Haven, Connecticut 06520 